\title{
La thalassémie
}

\author{
Hayley Merkeley MD MSc, Lauren Bolster MD
}

Citation : CMAJ 2020 October 13;192:E1210. doi : 10.1503/cmaj.191613-f

Voir la version anglaise de l'article ici : www.cmaj.ca/lookup/doi/10.1503/cmaj.191613

\section{La thalassémie peut être causée par des centaines de mutations héréditaires des gènes de l'hémoglobine. Le tableau clinique peut aller d'une présentation asymptomatique à la dépendance transfusionnelle.}

On ne connaît pas la prévalence de la thalassémie dans la population canadienne, mais il est probable qu'elle augmente avec les mouvements migratoires actuels ${ }^{1}$. Les personnes originaires de l'Afrique subsaharienne, de l'Asie du Sud-Est, des pays du bassin méditerranéen, du Moyen-Orient et du sous-continent indien sont particulièrement à risque, la prévalence allant de moins de $1 \%$ à $40 \%$ pour certains groupes ethniques ${ }^{1}$. En cas de soupçon clinique, l'origine ethnique du patient ne devrait pas décourager la prescription de tests de dépistage ${ }^{2,3}$.

2

La thalassémie est une cause à envisager en cas de microcytose inexpliquée, avec ou sans anémie.

Dans ses lignes directrices, l'Association canadienne des hémoglobinopathies (CanHaem) recommande que l'on procède à une formule sanguine complète et à des tests utilisant l'électrophorèse et la chromatographie liquide à haute performance chez les personnes qui présentent une microcytose inexpliquée sans avoir de carence en fer ${ }^{2}$. Cependant, l'électrophorèse de l'hémoglobine ne permet pas à elle seule d'écarter la possibilité de thalassémie. L'élévation du taux d'hémoglobine $A_{2}$ chez le fœtus, que le taux d'hémoglobine général soit élevé ou non, est généralement indicatrice du trait $\beta$-thalassémique et n'a pas besoin d'être confirmé en génétique. Dans le cas des a-thalassémies, le diagnostic dépend toujours des analyses génétiques, car les tests de dépistage des hémoglobinopathies donnent généralement des résultats normaux ${ }^{2,4}$. Le dépistage génétique est également recommandé pour les porteurs potentiels, avant la conception, et pour les personnes qui présentent des symptômes cliniques de la maladie.

\section{Chez les personnes atteintes de thalassémie, les intestins} absorbent davantage le fer.

Les personnes atteintes de thalassémie ne devraient pas prendre de suppléments de fer, à moins qu'elles aient une carence en fer attestée ${ }^{5}$. En présence d'un taux sérique de ferritine de plus de $300 \mu \mathrm{g} / \mathrm{L}$, il faut soupçonner un excès de fer et envoyer le patient consulter en hématologie, où l'on pourrait, selon le cas, procéder à un examen d'imagerie par résonance magnétique (IRM) et à une chélation ou une phlébotomie ${ }^{5}$.

\section{La thalassémie peut altérer les mesures de glycémie (diabète).} Étant donné la durée de vie réduite des globules rouges et la glycation anormale de l'hémoglobine $A$ associées à la thalassémie, les mesures du taux d'hémoglobine $A_{1 c}$ peuvent être faussées et ne sont pas recommandées chez les personnes atteintes, quelle que soit la gravité de la maladie $e^{6}$. Les personnes atteintes de thalassémie, qu'elles reçoivent ou non des transfusions, ont un risque accru de développer un diabète dû à un excès de fer, risque qui augmente avec l'âge ${ }^{5}$. Il vaut donc mieux mesurer la glycémie à jeun ou faire des épreuves d'hyperglycémie provoquée par voie orale ${ }^{2}$.

\section{5}

\section{Les personnes atteintes de}

thalassémie qui ont des symptômes doivent consulter des spécialistes.

Dans les cliniques multidisciplinaires de traitement de la thalassémie, on s'occupe de questions telles que les transfusions, la surveillance de l'excès de fer par IRM, la chélation du fer, les complications touchant les organes, la greffe de moelle osseuse et les essais cliniques ${ }^{1}$. Sur son site, CanHaem liste les centres spécialisés en la matière par provinces et propose une déclaration consensuelle sur les soins de la maladie - voir le www.canhaem.org.

\section{Références}

1. Sayani FA, Kwiatkowski JL. Increasing prevalence of thalassemia in America: implications for primary care. Ann Med 2015;47:592-604.

2. Belletrutti M, Bolster L, Corriveau-Bourque C, et al. Consensus statement of clinical care of patients with thalassemia in Canada, 2nd ed. In: Ezzat H, editor. The Canadian Haemoglobinopathy Association; 2017.. Accessible ici : www.canhaem.org/wp-content/uploads/2018/10/ consensus-statment-Thalassemia-Final.pdf (consulté le 25 novembre 2019). 
3. Viprakasit V, Ekwattanakit S. Clinical classification, screening and diagnosis of thalassemia. Hematol Oncol Clin North Am 2018;32:193-211.

4. Wilson RD, De Bie I, Armour C, et al. Joint SOGC-CCMG opinion for reproductive genetic carrier screening: an update for all Canadian providers of maternity and reproductive healthcare in the era of direct-to-consumer testing. J Obstet Gynaecol Can 2016;38:742-62.e3.

5. Guidelines for the management of non-transfusion dependent thalasseami. 2nd ed. Nicosia (Cyprus): Thalassemia International Society; 2017. Accessible ici : www.thalassemia. org/boduw/wp-content/uploads/2011/09/Guidelines-for-Mgmt -of-NTDT-TIF-2017.pdf (consulté le 2 mars 2020).

6. De Sanctis V, Soliman AT, Elsedfy H, et al. Growth and endocrine disorders in thalassemia: The international network on endocrine complications in thalassemia (I-CET) position statement and guidelines. Indian J Endocrinol Metab 2013; $17: 8-18$.

Intérêts concurrents : Aucun déclaré.

Cet article a été révisé par des pairs.

Affiliations : Département de médecine (Merkeley), Université de la Colombie-Britannique; Programme de la Colombie-Britannique pour les adultes atteints d'une hémoglobinopathie (Merkeley), Vancouver, C.-B.; Faculté de médecine et de médecine dentaire (Bolster), Université de l'Alberta, Edmonton, Alb.

Correspondance : Hayley Merkeley,

hmerkeley@providencehematology.com 